\title{
Contribution of Dissolved Oxygen to Methylene Blue Decomposition by Hybrid Advanced Oxidation Processes System
}

\author{
Heon Lee, ${ }^{1}$ Sung Hoon Park, ${ }^{1}$ Byung Hoon Kim, ${ }^{2}$ Sun-Jae Kim, ${ }^{3}$ Sang-Chai Kim, ${ }^{4}$ \\ Seong-Gyu Seo, ${ }^{5}$ and Sang-Chul Jung ${ }^{1}$ \\ ${ }^{1}$ Department of Environmental Engineering, Suncheon National University, Suncheon, Jeonnam 540-742, Republic of Korea \\ ${ }^{2}$ Department of Dental Materials, School of Dentistry, Chosun University, Gwangju 501-759, Republic of Korea \\ ${ }^{3}$ Faculty of Nanotechnology and Advanced Materials Engineering, Sejong University, Seoul 143-747, Republic of Korea \\ ${ }^{4}$ Department of Environmental Education, Mokpo National University, Muan, Jeonnam 534-729, Republic of Korea \\ ${ }^{5}$ Department of Civil \& Environmental Engineering, Chonnam National University, Yeosu, Jeonnam 550-749, Republic of Korea
}

Correspondence should be addressed to Sang-Chul Jung, jsc@sunchon.ac.kr

Received 1 March 2012; Revised 3 May 2012; Accepted 17 May 2012

Academic Editor: Manickavachagam Muruganandham

Copyright () 2012 Heon Lee et al. This is an open access article distributed under the Creative Commons Attribution License, which permits unrestricted use, distribution, and reproduction in any medium, provided the original work is properly cited.

Experimental results of photocatalysis under high dissolved oxygen (DO) concentration conditions are reported. Methylene blue was used as the organic pollutant to be degraded by a novel microwave/UV/DO/ $\mathrm{TiO}_{2}$ photocatalyst hybrid system. The degradation rate increased with $\mathrm{TiO}_{2}$ nanoparticle dosages and DO concentration. However, inhibition of photocatalysis due to bubbles produced by DO generator was also observed. When the DO generator was used to increase the DO concentration in the pollutant solution treated by the microwave-assisted $\mathrm{UV}-\mathrm{TiO}_{2}$ photocatalysis, the decomposition rate constant was highest among all the experimental conditions tested in this study. This result demonstrated that high concentration of DO can enhance the photocatalytic reaction rate by causing a synergistic effect of constituent techniques.

\section{Introduction}

Conventional wastewater treatment technologies have limitations hence demanding advanced research to tackle complex wastewater treatment. One of the promising technologies could be the more effective destruction by the use of advanced oxidation processes (AOPs) [1]. Application of photocatalyst in AOP industry effluents treatment has been investigated widely [2].

$\mathrm{TiO}_{2}$, a representative photocatalyst, has been successfully applied to the various photocatalytic reactions in a gas phase as well as in an aqueous phase [3]. Owing to its relatively high photocatalytic activity, biological and chemical stability, low cost, nontoxic nature, and long-term stability, $\mathrm{TiO}_{2}$ has been widely used as a photocatalyst [4-6].

Application of $\mathrm{TiO}_{2}$ photocatalyst in water treatment has recently been investigated widely [7]. There are still many problems yet to be solved, however, in application of
$\mathrm{TiO}_{2}$ photocatalyst in the treatment of non-biodegradable materials. First, photocatalysis has usually been used in air pollutants treatment because it is suitable for treatment of low-concentration pollutants. Concentrations of water pollutants, however, are much higher than those of air pollutants. Thus, their treatment by photocatalysis is difficult compared to that of air pollutants. Second, polluted water often contains mixture of hydrophilic and hydrophobic materials. Therefore, it is not easy for the pollutants to be adsorbed on the photocatalyst surface. Third, polluted water has high turbidity, hence, low transparency, hindering activation of photocatalysts by ultraviolet (UV) rays. Fourth, some materials are not easily degraded by photocatalysis only. Fifth, the amount of oxygen available for photocatalytic oxidation is very low in water compared to that in air. Due to these reasons, photocatalytic oxidation of water pollutants has not received large attention thus far. Recently, researches have been conducted actively to improve oxidative 
degradation performance by adding microwave irradiation as an effort to utilize $\mathrm{TiO}_{2}$ photocatalyst in water treatment more efficiently $[8,9]$.

On decomposition and mineralization, $\mathrm{TiO}_{2}$ photocatalysis consumes $\mathrm{O}_{2}$. In an earlier study on photocatalysis, this $\mathrm{O}_{2}$ consumption was found to be "photo-adsorption" of $\mathrm{O}_{2}$. The photoadsorption of $\mathrm{O}_{2}$ has been a general term of the consumption of $\mathrm{O}_{2}$ by photocatalytic reaction. Actually, photocatalytic reaction should proceed simultaneously by reduction and oxidation. In general photocatalysis, $\mathrm{O}_{2}$ in air is reduced to give $\mathrm{O}_{2}{ }^{-}$, while organic compounds are oxidized to form organic radicals which consume $\mathrm{O}_{2}$ as well for further oxidation [10-12].

In this study, the effect of dissolved oxygen (DO) on photocatalysis was investigated using a novel microwave/UV/ $\mathrm{DO} /$ photocatalyst hybrid system. A microwave-assisted electrodeless lamp is used as the UV source. The effect of microwave irradiation on photocatalytic reaction efficiency is investigated. To improve the pollutant removal rate, further $\mathrm{O}_{2}$ gas is added to the reaction and its effects on the reaction efficiency is investigated based on the experimental results. The role of each element technique and interaction among them are discussed.

\section{Experimental Procedure}

Figure 1 shows the schematic of the Microwave/UV-TiO ${ }_{2}$ experimental apparatus used in this study. Microwave radiation was emitted from a microwave system manufactured by Korea microwave instrument Co. Ltd. It consisted of a microwave generator (frequency, $2.45 \mathrm{GHz}$; maximal power, $1 \mathrm{~kW})$, a three-stub tuner, a power monitor, and a reaction cavity. Microwave radiation (actual power used, 200 $600 \mathrm{~W}$ ) used to irradiate the organic dye aqueous solution containing $\mathrm{TiO}_{2}$ particles was delivered through a waveguide. Microwave irradiation was continuous and its intensity was adjusted by connection to a power monitor. Optimal low reflection of the microwave radiation was achieved using the three-stub tuner. A stirrer was installed on the back side in the reaction cavity (Figure 1) to enhance the transfer of microwave. As the microwave-irradiated reactant solution is heated steadily, it was not possible to carry out experiments at constant temperature without a cooling system. In this study, the reactant solution was stored in a stainless steel beaker installed in a constant-temperature equipment. A pump in the DO generator was used to circulate the heated reactant solution through a cooling system to keep the reaction temperature constant at $298 \mathrm{~K}$.

In order to assess the effect of $\mathrm{DO}$ on $\mathrm{TiO}_{2}$ photocatalysis, DO concentration in the reaction solution was varied between 30 and 70 ppm using a DO generator (OxyLife Co.). $\mathrm{O}_{2}$ microbubbles were generated into the reaction solution inflow using a helix-type bubble generator installed in the DO generator tank. The DO concentration in the solution increased further while the solution passed devices with inner diameters smaller than that of the circulation pipe in the generator, due to increased flow velocity and friction coefficient. DO concentration was adjusted by using different
TABLE 1: Comparing the UV intensities radiated at different microwave intensities.

\begin{tabular}{llcc}
\hline \multirow{2}{*}{ Microwave intensity $(\mathrm{kW})$} & \multicolumn{3}{c}{ UV intensity $\left(\mathrm{W} / \mathrm{m}^{2}\right)$} \\
& UV-A & UV-B & UV-C \\
\hline 0.2 & 0.221 & 0.364 & 3.750 \\
0.4 & 0.577 & 0.922 & 5.570 \\
0.6 & 1.213 & 1.845 & 5.930 \\
\hline
\end{tabular}

combinations of the devices through which the solution passed.

$\mathrm{TiO}_{2}$ photocatalysts are excited by UV light, producing strong oxidants that can degrade organic compounds. Therefore, provision of UV is essential for a use of $\mathrm{TiO}_{2}$ photocatalysts. Typical UV lamps, however, have metal electrodes, which prevent them from being used in the microwave-irradiation equipment. Therefore, a doubletube-type microwave discharge electrodeless lamp (170 mm length, $44 \mathrm{~mm}$ inner diameter, $60 \mathrm{~mm}$ outer diameter, hereafter MDEL) which emits UV upon the irradiation of microwave was developed in this study. It was made of quartz to maximize the reaction efficiency. Small amount of mercury was doped between the tubes inside the double-tube UV lamp that was kept vacuumed. The lamp used in this study is UV-C-type lamp although little amount of UV-A and UV-B wavelength lights are emitted as well. Figure 2 shows the MDEL emitting UV light upon microwave irradiation in the microwave cavity. Another measurement made in this study was the irradiance $\left(\mathrm{W} / \mathrm{m}^{2}\right)$ measured at three different UV wavelength ranges that are called UV-A, UV-B, and UV$C$ with the wavelength range of $315 \sim 400 \mathrm{~nm}, 280 \sim 315 \mathrm{~nm}$, and $220 \sim 280 \mathrm{~nm}$, respectively. A UV radiometer (HD21022, Delta OHM) was used to measure the irradiance. Table 1 summarizes the UV irradiance measured with three different microwave intensities. The UV irradiances at all three wavelength ranges were shown to increase with the microwave intensity.

The photocatalyst was Degussa P-25 $\mathrm{TiO}_{2}$ (specific surface area $53 \mathrm{~m}^{2} \mathrm{~g}^{-1}$ measured by the BET method, particle size $20 \sim 30 \mathrm{~nm}$ by TEM, composition $83 \%$ anatase and $17 \%$ rutile by X-ray diffraction). In this study, the photocatalytic activity of $\mathrm{TiO}_{2}$ particle was investigated with the photocatalytic decomposition of methylene blue (referred to as $\mathrm{MB}$ hereafter) in its aqueous solution. High-puritygrade $\mathrm{MB}$ was purchased from Jin Chem. Co. Ltd. Initial concentration of $\mathrm{MB}$ was about $3.0 \times 10^{-5} \mathrm{~mol} / \mathrm{liter}$, and $3,000 \mathrm{~mL}$ of solution was circulated into the quartz reactor tube ( $230 \mathrm{~mm}$ length, $40 \mathrm{~mm}$ diameter) with a flow rate of $500 \mathrm{cc} / \mathrm{min}$.

The solution contained in a stainless steel beaker located in a cooling bath was circulated through the reactor and DO generator for 60 minutes to maintain the initial reactant concentration at constant. P25 $(0.1 \sim 0.3 \mathrm{~g})$ was injected into the beaker, and then the solution was circulated for another 5 minutes to disperse P25 throughout the entire system. $\mathrm{TiO}_{2}$ photocatalysis was performed by irradiating microwave and UV using the microwave generator $(0.2 / 0.4 / 0.6 \mathrm{Kw})$. The 


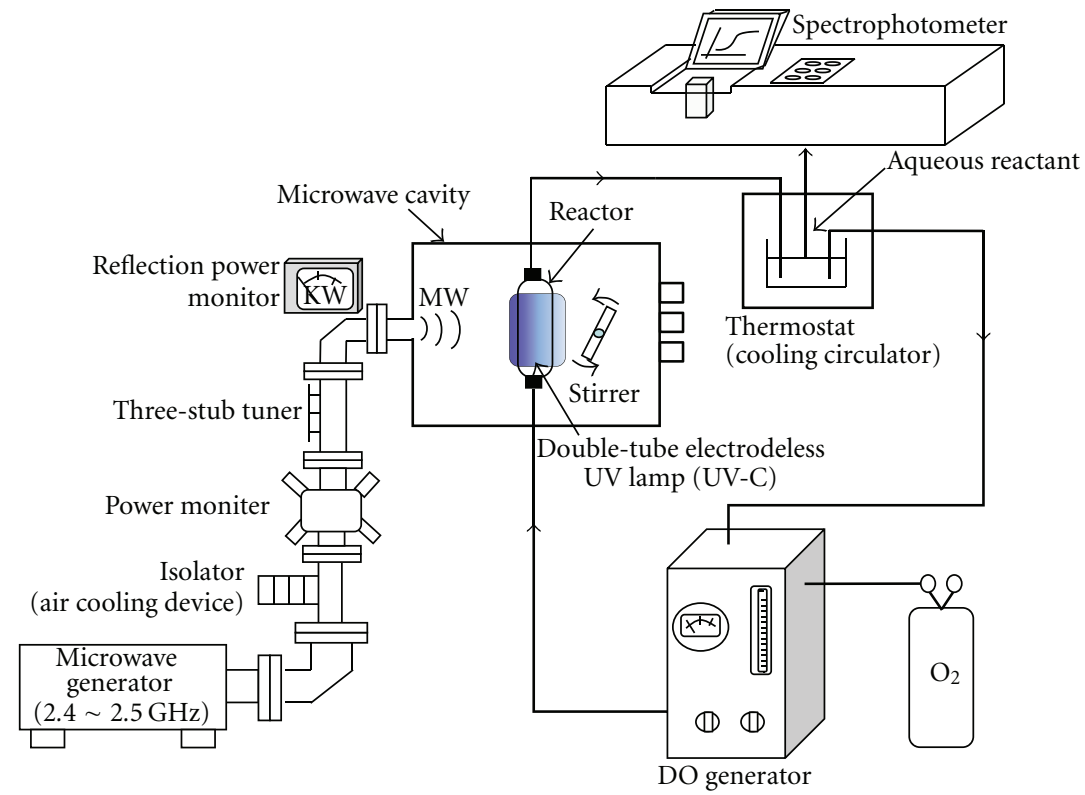

FIgURE 1: Schematics of the microwave/UV/DO $\mathrm{TiO}_{2}$ photocatalytic degradation system.

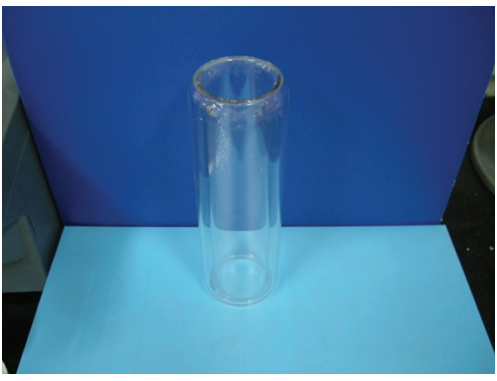

(a)

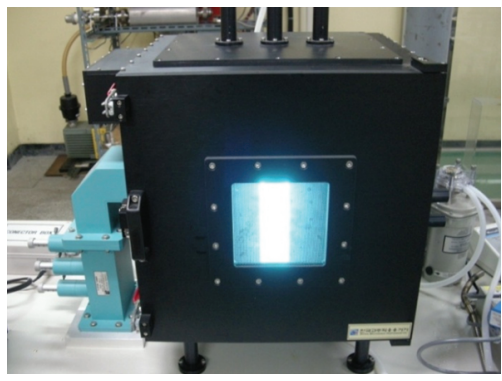

(b)

FIgURE 2: Snapshot of the double-tube-type MDEL (a) and microwave-discharged lamp set in the microwave oven (b).

solution samples taken at predefined time steps were centrifuged at 2,000 rpm for 30 minutes. Then, the absorbance of supernatant was measured using a spectrophotometer (UV-1601, Shimadzu) at $665 \mathrm{~nm}$ to obtain the reaction rate. The solution samples used for absorbance measurements were injected back into the reaction system.

\section{Result and Discussion}

3.1. Effect of $\mathrm{TiO}_{2}$ Particle Dosages. Figure 3 shows the results of decomposition experiments of $\mathrm{MB}$ obtained at different $\mathrm{TiO}_{2}$ particle dosages. The microwave intensity was $0.4 \mathrm{~kW}$, and the $\mathrm{DO}$ concentration was $70 \mathrm{ppm}$. Addition of a larger amount of $\mathrm{TiO}_{2}$ particle resulted in a higher decomposition rate. Certain amount of $\mathrm{MB}$ was shown to be decomposed by MDEL and high concentration of $\mathrm{DO}$ without the aid of $\mathrm{TiO}_{2}$ photocatalytic reaction. The plots for the four cases were all fitted well by linear line, which indicates that decomposition of $\mathrm{MB}$ in the presence of $\mathrm{TiO}_{2}$ catalyst can be approximated by a pseudo-first-order reaction model:

$$
\frac{C}{C_{0}}=\exp (-K t)
$$

where $C$ is the $\mathrm{MB}$ concentration at time $t, C_{0}$ the initial concentration, and $K$ the overall rate constant. The overall rate constant $K$ is determined as the slope of the line in Figure 3 by regression analysis. It is clearly shown in this figure that the degradation rate increases with the amount of $\mathrm{TiO}_{2}$ particle dosages.

3.2. Relationship between the Degradation Rate and DO Levels. The rates of $\mathrm{MB}$ degradation by $\mathrm{TiO}_{2}$ photocatalytic reaction 


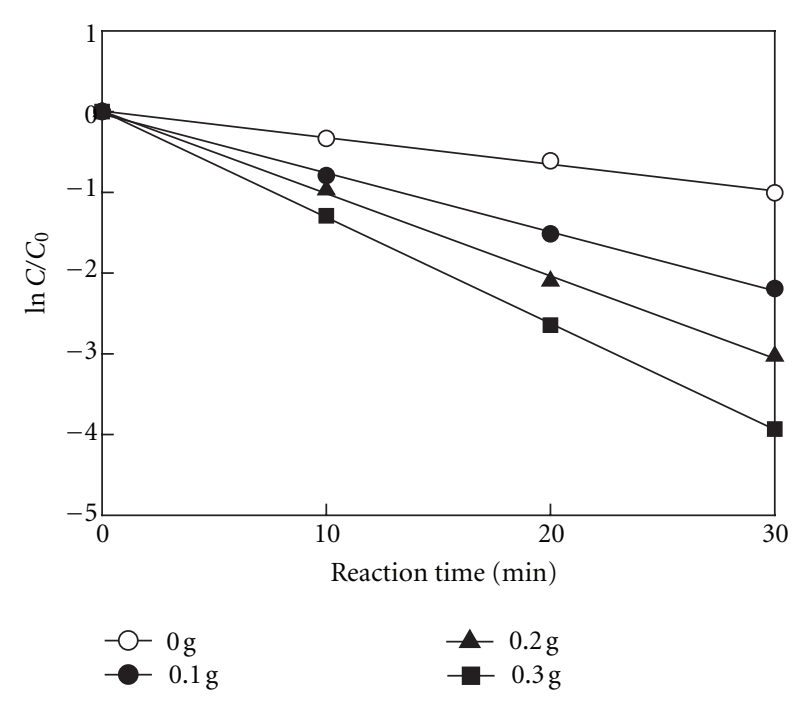

Figure 3: Effect of $\mathrm{TiO}_{2}$ particle dosages for decomposition of methylene blue in aqueous solutions.

measured at different DO concentration levels ( $5 \sim 70 \mathrm{ppm})$ are shown in Figure 4. The average DO concentration of the as-prepared $\mathrm{MB}$ solution without using $\mathrm{DO}$ generator was about $5 \mathrm{ppm}$. All experiments were carried out under the microwave intensity of $0.4 \mathrm{~kW}$ and the $\mathrm{TiO}_{2}$ particle dosage of $0.2 \mathrm{~g}$. When the DO generator was used to adjust the DO concentration between 30 and $70 \mathrm{ppm}$, the degradation rate was shown to increase with DO concentration. However, the degradation rates measured at $30 \mathrm{ppm}$ and $50 \mathrm{ppm}$ were lower than that measured without using the DO generator (at $5 \mathrm{ppm}$ ). This result may be attributed to the inhibition effect of the bubbles. In this study, highpressure oxygen gas was injected into the DO generator in which the oxygen bubbles were produced by the helixtype bubble generator. In general, photocatalysis is a surface reaction. Bubbles produced by the DO generator may be attached on the photocatalyst surface inhibiting the surface reaction. Nevertheless, when DO concentration was high enough $(70 \mathrm{ppm})$, the degradation rate was higher than that measured without using the DO generator. Moreover, a higher DO concentration resulted in a higher degradation rate among the experiments in which a DO generator was used. This result indicates that dissolved oxygen promotes the photocatalytic decomposition of MB.

The effect of dissolved oxygen can be explained as follows. UV irradiated on $\mathrm{TiO}_{2}$ photocatalysts causes excitation, leading to movement of electrons in the valance band towards the conduction band. If the reaction does not follow immediately, recombination occurs. By playing a role as electron capturers, oxygen molecules can slow down the recombination rate and help produce peroxide and hydroperoxy radicals and therefore enhance the degradation reaction rate [13].

The results of this study showed that the use of a DO generator may result in inhibition of photocatalysis due to attachment of bubbles on the catalyst surface.

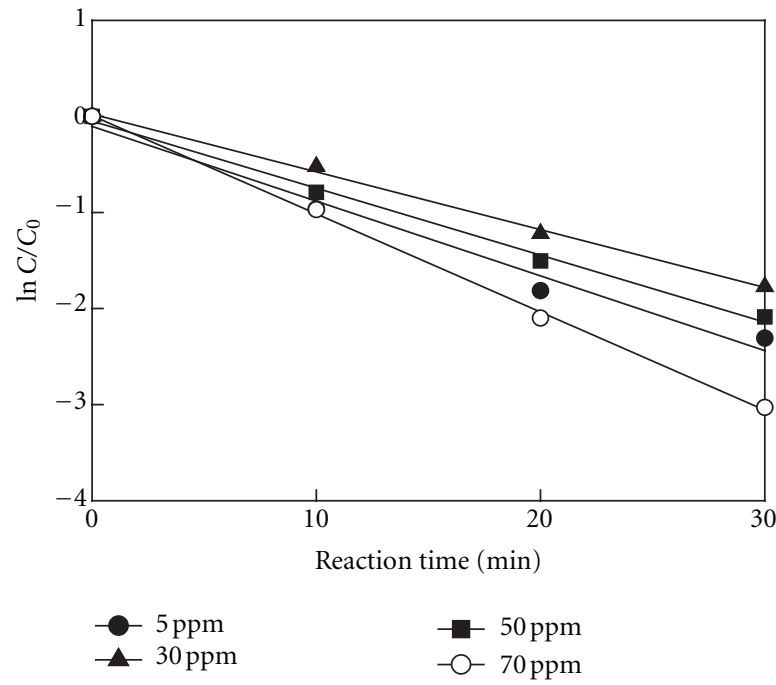

FIGURE 4: Degradation rate of methylene blue measured at various dissolved oxygen levels.

Therefore, when a DO generator is applied for enhancing the photocatalysis rate, high concentration of $\mathrm{DO}$, enough to compensate the side effect of bubble attachment, is required.

3.3. Microwave Effects in Photocatalytic Oxidation Reactions. $\mathrm{MB}$ decomposition experiments were carried out at different microwave intensity levels to evaluate the effect of microwave intensity on photocatalysis. The results are shown in Figure 5 as a function of microwave intensity. The experiments were carried out with the $0.2 \mathrm{~g} \mathrm{TiO}_{2}$ particle at $70 \mathrm{ppm}$ DO concentration. Three different microwave powers were used: $0.2,0.4$, and $0.6 \mathrm{~kW}$. It is clearly shown in this figure that the degradation rate increases with microwave intensity. Microwave causes polar reactant molecules to vibrate million times a second. Microwave has thermal and nonthermal effects. The thermal effect denotes the increase in the chemical reaction rate due to selective, fast, uniform increase of temperature by microwave radiation. The nonthermal effect represents the promotion of the chemical reaction resulting from increased molecular movement and collision frequency. In this study, temperature was controlled at constant to exclude the thermal effect. Therefore, the increase in the degradation rate by the microwave irradiation is believed to result from the resulting UV radiation and the non-thermal effect of microwave.

In this study, a short wavelength electromagnetic wave UV is emitted by MDEL upon the irradiation of microwave. Therefore, the intensity of UV increases with the microwave power. UV, which carries intense energy, is used for exciting photocatalyst. It can also contribute to degrading $\mathrm{MB}$ directly. It was not possible to figure out the detailed mechanism how microwave took part in the degradation of MB. Nevertheless, it can be inferred from the experimental result, which showed higher degradation efficiency at higher 


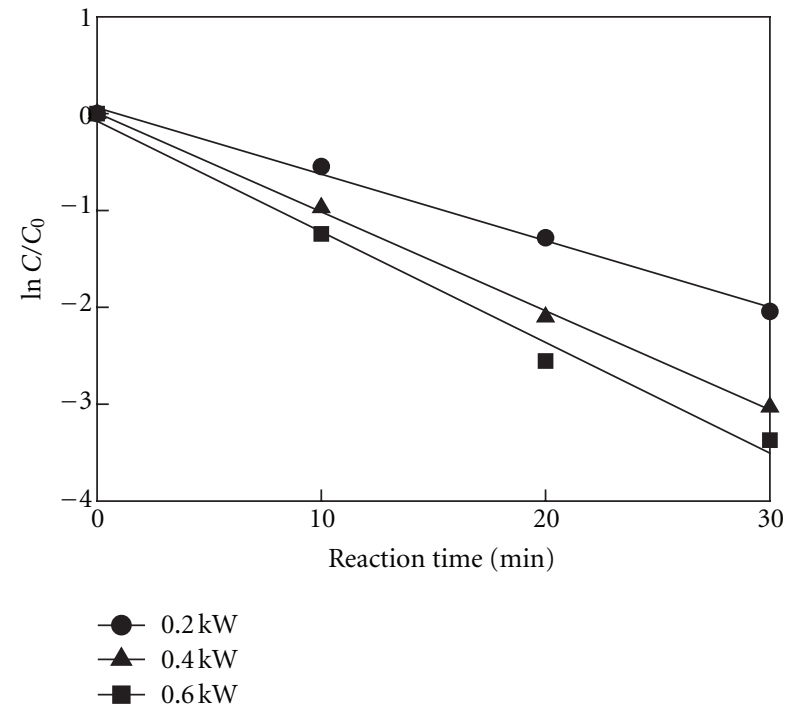

FIgURE 5: Effect of microwave intensity for decomposition of methylene blue in aqueous solution.

microwave intensity, that microwave contributed to degradation of MB indirectly by increasing UV intensity. The thermal and non-thermal effects of microwave are also presumed to have contributed directly to the degradation reaction.

3.4. Comparison of the Effects of the Constituent Techniques. This study was aimed at enhancing the photocatalytic degradation rate of $\mathrm{MB}$ by adding microwave and high-concentration DO. To evaluate the effect of each constituent technique, different sets of combinations of microwave, DO, UV, and photocatalyst were used for degradation of organic dye. Figure 6 shows the degradation rate constants obtained at different conditions of the microwave/UV/DO/photocatalyst hybrid process system. The amount of reaction solution of $3,000 \mathrm{~mL}, \mathrm{DO}$ concentration of $70 \mathrm{ppm}$, and the microwave intensity of $0.4 \mathrm{~kW}$ were used for all experiments. The results of six different experiments are compared in Figure 6: microwave irradiation only $(\mathrm{M})$, DO generator only (D), microwave irradiation on top of the use of MDEL (MU), addition of DO generator to MU (MUD), microwave-assisted UV$\mathrm{TiO}_{2}$ photocatalysis by MDEL (MUP), and addition of DO generator to MUP (MUPD).

When only microwave was applied (M), MB was seldom degraded because the thermal effect was excluded in this study by maintaining the temperature of the solution at $298 \mathrm{~K}$. On the other hand, when only DO generator was used (D) to adjust the DO concentration at $70 \mathrm{ppm}$, certain level of degradation reaction was detected. When microwave and MDEL were used together (MU), the degradation rate was significantly higher. As the DO generator was added to MU (MUD) to increase the DO concentration, the degradation rate was reduced. This result can be attributed to the inhibition effect of the bubbles produced by DO generator as was explained above. For the microwave-assisted UV$\mathrm{TiO}_{2}$ photocatalysis (MUP), the decomposition rate constant

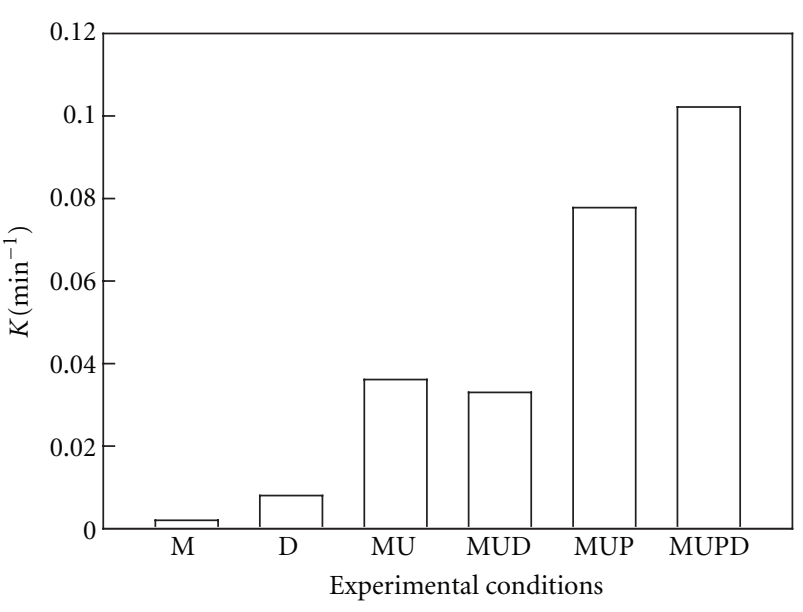

FIGURE 6: Rate constants obtained under different experimental conditions.

was very high. When the DO generator was added to MUP (MUPD), the rate constant was even higher. This result demonstrates that high concentration of DO can enhance the photocatalytic reaction rate by causing a synergistic effect of constituent techniques.

The results of this study show that combining elemental techniques, which are not very efficient when they are used exclusively, induce a synergistic effect. The objective of this study was to promote the photocatalytic reaction by adding microwave irradiation, which was expected to activate pollutant and photocatalyst, and high-concentration DO, as an auxiliary oxidant, to the conventional photocatalysis system. According to the experimental results of this study, the influence of microwave on organic dye and photocatalyst was minimal, whereas high-concentration DO was shown to contribute to production of activation species that participate in the degradation reaction. Inhibition of photocatalysis due to bubbles produced by DO generator was also observed, indicating that care should be taken to find an optimal hybrid process for practical use.

The above-mentioned explanations, however, cannot be proven with the limited experimental data obtained in this study. More precise and quantitative analyses on the elemental techniques tested in this study are required in the future for more reliable evaluation and application of them.

\section{Conclusions}

The following conclusions were inferred from the results of advanced oxidation of $\mathrm{MB}$ by novel microwave/UV/DO/ $\mathrm{TiO}_{2}$ photocatalysts hybrid process system.

(1) The degradation rate increased with $\mathrm{TiO}_{2}$ particle dosages, microwave intensity and DO concentration.

(2) When a DO generator was used to adjust the DO concentration between 30 and $70 \mathrm{ppm}$, the degradation 
rate was shown to increase with DO concentration. However, the degradation rates measured at $30 \mathrm{ppm}$ and $50 \mathrm{ppm}$ were lower than that measured without using the DO generator (at $5 \mathrm{ppm}$ ).

(3) The result demonstrates that high concentration of DO can enhance the photocatalytic reaction rate by causing a synergistic effect of constituent techniques.

(4) The results of this study showed that the use of a DO generator may result in inhibition of photocatalysis due to attachment of bubbles on the catalyst surface. Therefore, when a DO generator is applied for enhancing the photocatalysis rate, high concentration of DO, enough to compensate the side effect of bubble attachment, is required.

\section{Acknowledgment}

This research was supported by the Basic Science Research Program through the National Research Foundation of Korea (NRF) funded by the Ministry of Education, Science and Technology (2010-0007412 and 2011-0016699).

\section{References}

[1] W. F. Jardim, S. G. Moraes, and M. M. K. Takiyama, "Photocatalytic degradation of aromatic chlorinated compounds using $\mathrm{TiO}_{2}$ : toxicity of intermediates," Water Research, vol. 31, no. 7, pp. 1728-1732, 1997.

[2] P. Saritha, C. Aparna, V. Himabindu, and Y. Anjaneyulu, "Comparison of various advanced oxidation processes for the degradation of 4-chloro-2 nitrophenol," Journal of Hazardous Materials, vol. 149, no. 3, pp. 609-614, 2007.

[3] R. W. Matthews, "Photo-oxidation of organic material in aqueous suspensions of titanium dioxide," Water Research, vol. 20, no. 5, pp. 569-578, 1986.

[4] A. Dixit, A. J. Tirpude, A. K. Mungray, and M. Chakraborty, "Degradation of 2, 4 DCP by sequential biological-advanced oxidation process using $\mathrm{UASB}$ and $\mathrm{UV} / \mathrm{TiO}_{2} / \mathrm{H}_{2} \mathrm{O}_{2}$," Desalination, vol. 272, no. 1-3, pp. 265-269, 2011.

[5] S. C. Jung, S. J. Kim, N. Imaishi, and Y. I. Cho, "Effect of $\mathrm{TiO}_{2}$ thin film thickness and specific surface area by low-pressure metal-organic chemical vapor deposition on photocatalytic activities," Applied Catalysis B, vol. 55, no. 4, pp. 253-257, 2005.

[6] S. C. Jung, "Photocatalytic activities and specific surface area of $\mathrm{TiO}_{2}$ films prepared by CVD and sol-gel method," Korean Journal of Chemical Engineering, vol. 25, no. 2, pp. 364-367, 2008.

[7] M. Harir, A. Gaspar, B. Kanawati et al., "Photocatalytic reactions of imazamox at $\mathrm{TiO}_{2}, \mathrm{H}_{2} \mathrm{O}_{2}$ and $\mathrm{TiO}_{2} / \mathrm{H}_{2} \mathrm{O}_{2}$ in water interfaces: kinetic and photoproducts study," Applied Catalysis B, vol. 84, no. 3-4, pp. 524-532, 2008.

[8] G. Zhanqi, Y. Shaogui, T. Na, and S. Cheng, "Microwave assisted rapid and complete degradation of atrazine using $\mathrm{TiO}_{2}$ nanotube photocatalyst suspensions," Journal of Hazardous Materials, vol. 145, no. 3, pp. 424-430, 2007.

[9] S. C. Jung, "The microwave-assisted photo-catalytic degradation of organic dyes," Water Science and Technology, vol. 63, no. 7, pp. 1491-1498, 2011.
[10] T. Hirakawa, C. Koga, N. Negishi, K. Takeuchi, and S. Matsuzawa, "An approach to elucidating photocatalytic reaction mechanisms by monitoring dissolved oxygen: effect of $\mathrm{H}_{2} \mathrm{O}_{2}$ on photocatalysis," Applied Catalysis B, vol. 87, no. 1-2, pp. 46$55,2009$.

[11] H. C. Liang, X. Z. Li, Y. H. Yang, and K. H. Sze, "Effects of dissolved oxygen, $\mathrm{pH}$, and anions on the 2,3-dichlorophenol degradation by photocatalytic reaction with anodic $\mathrm{TiO}_{2}$ nanotube films," Chemosphere, vol. 73, no. 5, pp. 805-812, 2008.

[12] C. C. Lo, C. W. Huang, C. H. Liao, and J. C. S. Wu, "Novel twin reactor for separate evolution of hydrogen and oxygen in photocatalytic water splitting," International Journal of Hydrogen Energy, vol. 35, no. 4, pp. 1523-1529, 2010.

[13] M. R. Hoffmann, S. T. Martin, W. Choi, and D. W. Bahnemann, "Environmental applications of semiconductor photocatalysis," Chemical Reviews, vol. 95, no. 1, pp. 69-96, 1995. 


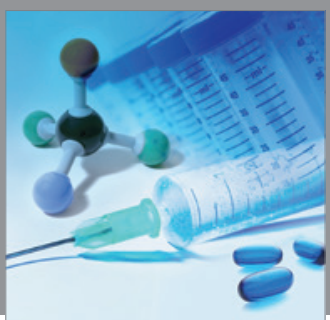

International Journal of

Medicinal Chemistry

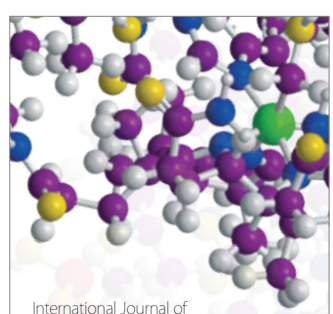

Carbohydrate Chemistry

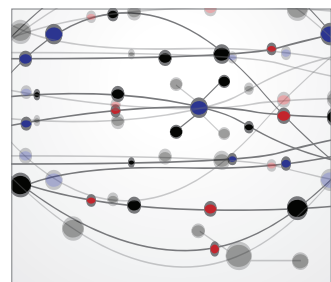

The Scientific World Journal
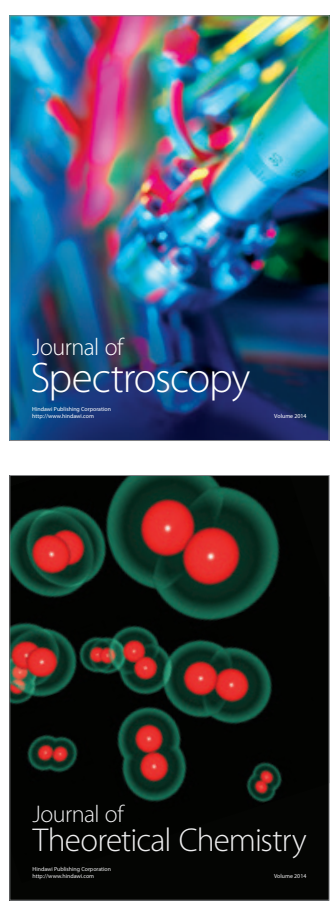
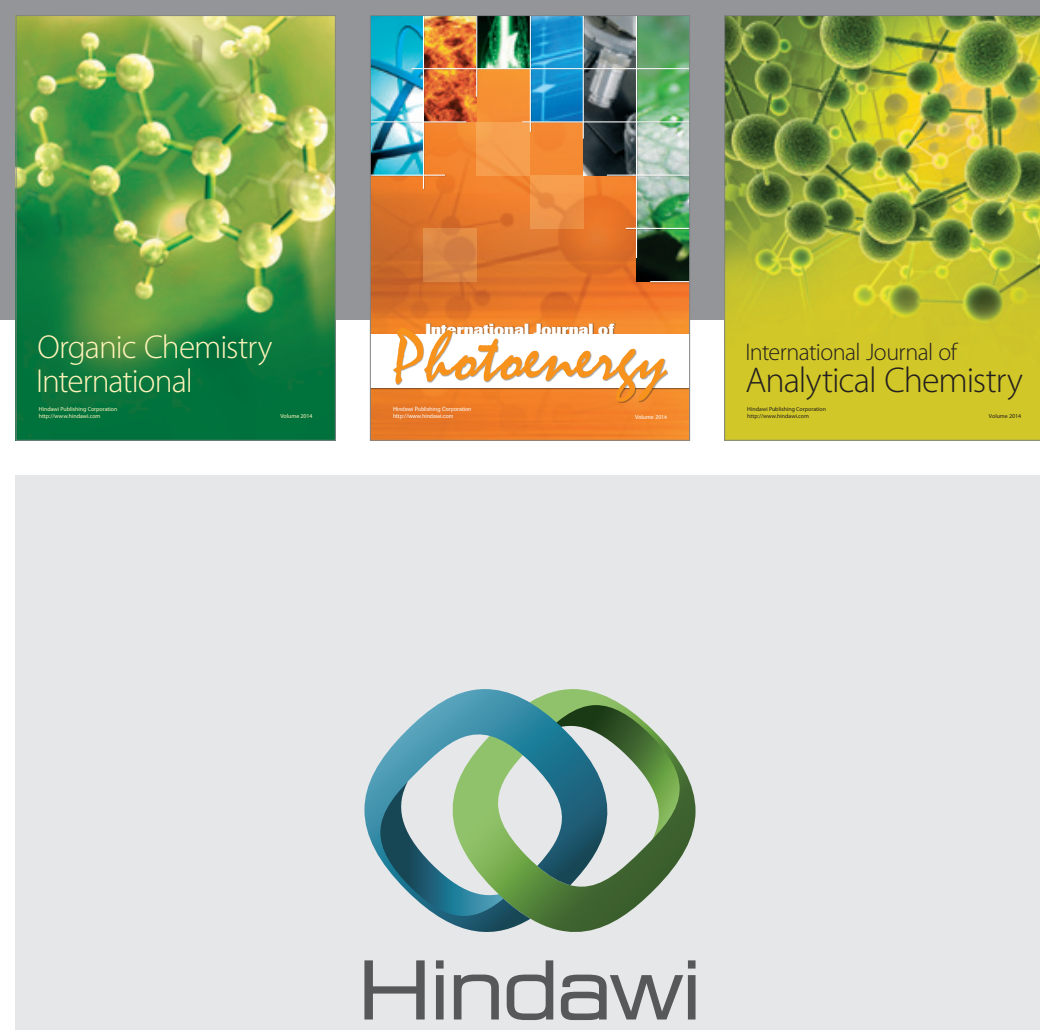

Submit your manuscripts at

http://www.hindawi.com
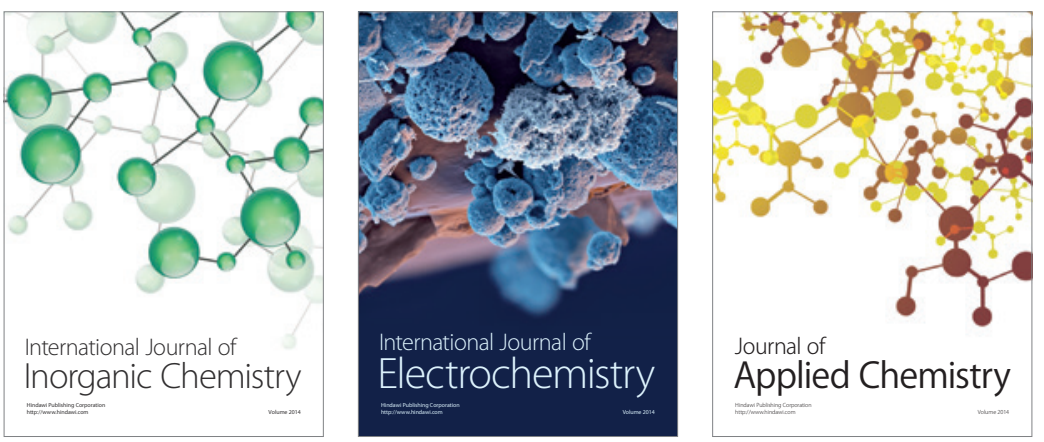

Journal of

Applied Chemistry
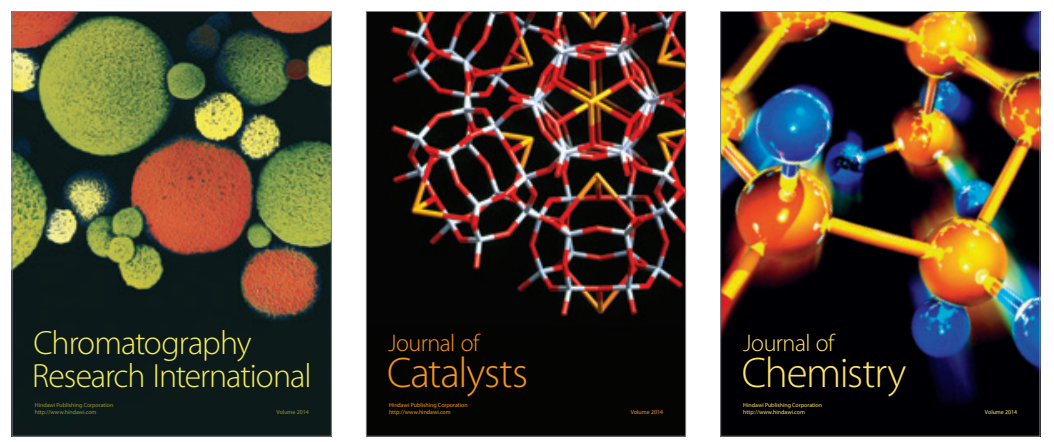
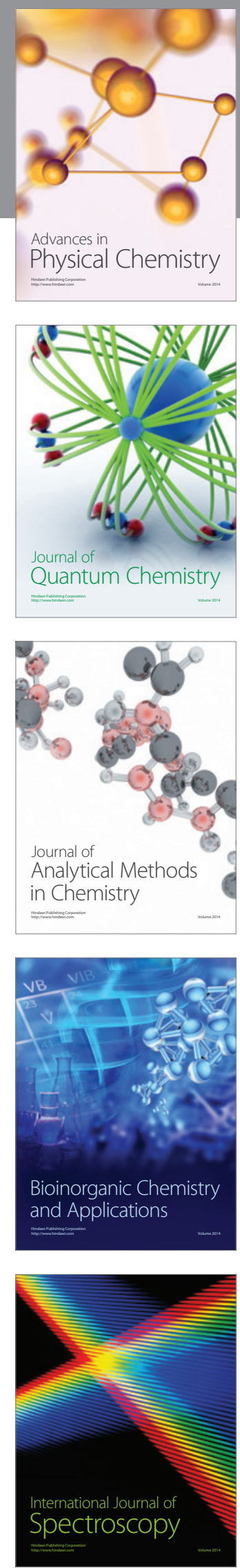